\title{
HARMONIC NONHOLOMORPHIC MAPS FROM A SURFACE TO A SPHERE
}

\author{
LUC LEMAIRE ${ }^{1}$
}

\begin{abstract}
For every $p$ and $\mathscr{D}$ such that $|\mathscr{}| \leqslant p-1$, there exist a Riemann surface of genus $p$ and a harmonic nonholomorphic map of degree D from that surface to the sphere. For some of the degrees, a holomorphic map exists for the same Riemann surface.
\end{abstract}

Introduction. Let $M, g$ and $N, h$ be compact Riemannian $C^{\infty}$ manifolds. A map $\varphi \in C^{\infty}(M, N)$ is said to be harmonic iff it is a critical point of the energy function $E(\varphi)=\frac{1}{2} \int_{M}|d \varphi|^{2} v_{g}[1]$.

In this paper, we suppose that $M$ and $N$ are compact orientable surfaces, whose genera are denoted by $p$ and $q$, and study the problem of the existence of harmonic representatives in the different homotopy classes of maps from $M$ to $N$.

When $q \neq 0$, we know ([4] and [5]) that, for any metrics $g$ and $h$ on $M$ and $N$, every homotopy class contains a harmonic map.

When $q=0$ (i.e. $N$ is the sphere $S^{2}$ ), the homotopy classes are parametrized by the degree $\mathscr{D}$ of the maps, and J. Eells and J. Wood [2] have shown that every harmonic map whose degree satisfies $|\mathscr{D}| \geqslant p$ is holomorphic or antiholomorphic with respect to the complex structures associated with $g$ and $h$. Using this fact, they solved the existence question for these values of the degree, obtaining some existence and nonexistence results.

In this paper, we shall consider the case $|\mathscr{D}| \leqslant p-1$, and show that for every such $p$ and $\mathscr{D}$, there exist a surface $M$ of genus $p$ and a harmonic nonholomorphic map $\varphi$ of degree $\mathscr{D}$ from $M$ to $S^{2}(\S 1)$. This result was already obtained in [5] for $1 \leqslant|\mathscr{D}| \leqslant p / 2$, but the method used there does not seem to extend to the other values of $\mathscr{D}$.

We then exhibit some homotopy classes containing two harmonic maps, only one of them being holomorphic (\$2) and conclude by an example of a continuous family of harmonic maps which are not isometrically or conformally equivalent (\$3).

1. Harmonic nonholomorphic maps. Recall ([1], [8]) that the harmonic maps between two surfaces $M$ and $N$ are preserved by a conformal change of

Received by the editors June 10, 1977.

AMS (MOS) subject classifications (1970). Primary 58C10, 58E15; Secondary 30A26, 30A68, $53 \mathrm{C} 20$.

Key words and phrases. Harmonic maps, holomorphic map.

${ }^{1}$ Chargé de recherches au Fonds National Belge de la Recherche Scientifique. 
metric on $M$. Therefore, they only depend on the complex structure associated to the metric $g$.

(1.1) THEOREM. For every $p \geqslant 1$ and $\mathscr{D}$ such that $|\mathscr{D}| \leqslant p-1$, there exist a Riemann surface $M$ of genus $p$ and a metric $h$ on the sphere $S^{2}$ such that there is a harmonic nonholomorphic (and nonantiholomorphic) map of degree $\mathscr{D}$ from $M$ to $S^{2}$. That map is not a minimum of $E$ in the class of maps of degree $\mathscr{D}$.

REMARK. This theorem provides a partial existence result for $|\mathscr{D}| \leqslant p-1$ and also shows that the result of J. Eells and J. Wood does not extend to these degrees.

The last assertion of Theorem 1.1 was proven in [4] and [5]. The first one will be a consequence of Propositions (1.2)-(1.4) and (1.9) concerning different values of $\mathscr{D}$. As a change of orientation on $M$ will change the sign of $\mathscr{Q}$, we can suppose from now on that $0 \leqslant \mathscr{D} \leqslant p-1$.

First case. $\mathscr{D}=1$.

(1.2) Proposition. Let $M$ be a surface of even genus $\geqslant 2$, symmetric with respect to three orthogonal lines and $N$ a sphere with a metric possessing the same symmetry properties. Then there is a harmonic nonholomorphic map of degree 1 from $M$ to $N$.

(1.3) Proposition. Let $M$ be a surface of genus $p \geqslant 2$ which can be represented as a sphere with handles symmetric with respect to an equator and $p$ meridians forming angles $\Pi / p$. Let $N$ be a surface of revolution of genus 0 , symmetric with respect to its equator. Then there is a harmonic map from $M$ to $N$ of all degrees smaller or equal to $p / 2$.

These two propositions were proven in [4], [5].

Second case. $2 \leqslant \mathscr{D} \leqslant p-1$.

(1.4) Proposition. Let $N$ be a surface of genus 0 symmetric with respect to three orthogonal lines. For any $p \geqslant 3$ and $\mathscr{D}$ such that $2 \leqslant \mathscr{D} \leqslant p-1$, there exist a surface $M$ of genus $p$ and a harmonic nonholomorphic map of degree $\mathscr{Q}$ from $M$ to $N$.

Proof. It is known [8] that in dimension two the composition of a holomorphic map with a harmonic one is harmonic (this is a direct consequence of the composition law $[1, I, 5]$ and the fact that a holomorphic map between surfaces is weakly conformal and harmonic [5]). As Proposition 1.2 asserts the existence of a harmonic nonholomorphic map of degree one from a surface $\bar{M}$ of genus two to $S^{2}$ and as the composition of a holomorphic covering map with a nonholomorphic map is nonholomorphic, Proposition 1.4 will be a consequence of the following:

(1.5) LEMMA. Let $\bar{M}$ be a Riemann surface of genus 2 and $p$ and $\mathscr{D}$ two integers such that $2 \leqslant \mathscr{D} \leqslant p-1$. There exists a Riemann surface of genus $p$ which is $a$ D -sheeted covering of $\bar{M}$ with holomorphic projection. 
Proof. It is sufficient to build a topological surface $M$ which is a branched covering of $\bar{M}$. Indeed, it will then admit a unique complex structure for which the projection is holomorphic [3].

Let us recall that if $M$ is a $\mathscr{D}$-sheeted covering of $\bar{M}$, their genera $p$ and $\bar{p}$ are related by Hurwitz' formula

$$
2-2 p+r=\mathscr{D}(2-2 \bar{p})
$$

where $r$ is the ramification index, i.e. the sum on all branch points of the local degree of the projection minus one. Here we have $\bar{p}=2$, and (1.6) becomes

$$
r=-2 \mathscr{D}-2+2 p
$$

Since $r$ must be nonnegative, a necessary condition for the existence of $M$ is therefore $\mathscr{D} \leqslant p-1$, which is what we have supposed. $p$ and $\mathscr{D}$ being given, we shall now build the covering, using a joining process indicated by H. Rauch.

We consider $\mathscr{D}$ copies of $\bar{M}$. With any two of these copies, we can form a 2-sheeted covering by joining them crosswise along a line (by this we mean that we choose on both copies the "same" line-i.e. two lines such that one is the copy of the other-make a slit in each surface along the line and join them crosswise along the slit). That line could be closed, but not separating, in which case there is no ramification, or it could be a segment with two endpoints and induce a ramification index of two. We can also join two copies by more than one segment, which gives a higher ramification index. Having joined two copies of $\bar{M}$, we can then add the others in a similar way. The idea is then to choose the number of segments in order to obtain a ramification index $r$ that will impose the right genus $p$ through (1.7).

If for the given $p, r / 2=\mathscr{D}-1$, we use exactly $r / 2$ segments to join the $\mathscr{D}$ copies. If $r / 2>\mathscr{D}-1$, we join two of the copies along $r / 2-\mathscr{D}+2$ segments and add the others using one segment at a time. If, finally, $r / 2<\mathscr{D}-1$, we use $r / 2$ segments and $\mathscr{D}-1-r / 2$ closed lines. In all cases, we obtain a $\mathscr{D}$-sheeted covering with ramification index $r$ and hence genus $p$.

(1.8) REMARK. In the case $\mathscr{D}=p-1$, we can give a more geometric representation of $M$, suggested by E. Calabi.

Let $M$ be a surface of genus $p$, invariant by a rotation of order $p-1$, as represented on Figure 1 in the case $p=5$. The quotient of $M$ by the rotation is a surface $\bar{M}$ of genus 2 . We can then endow $\bar{M}$ with the required complex structure and lift it to the original surface. This is an unbranched covering $(r=0)$.

Third case. $\mathcal{Q}=0$.

When $\mathscr{D}=0$, we obtain a stronger result:

(1.9) Proposition. Let $M$ be a surface of genus greater or equal to one and $N$ a surface of genus zero, with arbitrary metrics. Then there exists a harmonic nonholomorphic map of degree zero from $M$ to $N$. 


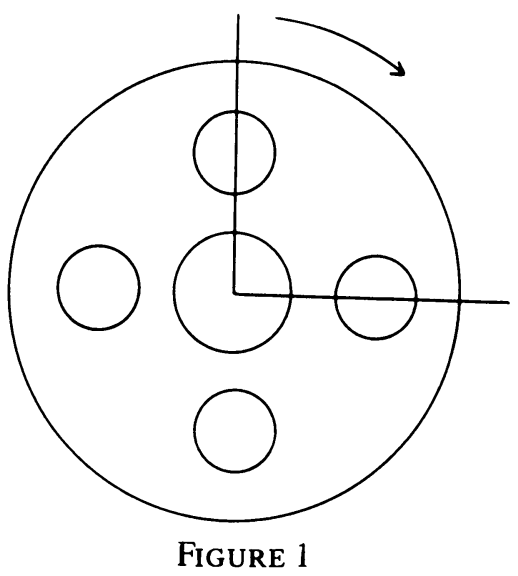

PRoof. Consider a circle $S^{1}$. By [1, I,4,D] there exists a surjective harmonic map from $M$ to $S^{1}$. On the other hand, the surface $N$ admits a closed geodesic, realized by a totally geodesic map from $S^{1}$ to $N$. The composition of these two maps is harmonic [1, I,5,A]. Since its image is one dimensional, it is not holomorphic.

(1.10) RemarK. For certain metrics on $M$ and $N$, one can in fact construct harmonic nonholomorphic maps of degree zero which are also surjective.

When $M$ is a flat orthogonal torus and $N$ the canonical sphere, such a map was constructed by R. T. Smith [6].

For a higher $p$, it is then sufficient to build a branched covering of genus $p$ of the torus. (1.6) becomes in this case $r=2 p-2$ and we obtain a covering by joining two or more copies of the torus along $p-1$ segments.

As in remark (1.8), a more geometric representation of such a covering (with $p$ sheets) can be obtained by starting with a surface $M$ of genus $p$ invariant by a rotation of order $p$ with two fixed points and taking the quotient of $M$ by the rotation. The ramification is then reduced to two branch points of order $p-1$.

2. Homotopy classes with two critical values of the energy. We now show that the same nontrivial homotopy class can contain two harmonic maps, one of them being holomorphic, the other not. The first is a minimum of $E$ in the class and the second has a greater energy.

(2.1) Proposition. For every $p \geqslant 3$, there exist a surface $M$ of genus $p$ and $a$ nontrivial homotopy class of maps from $M$ to $S^{2}$ containing two harmonic maps, one holomorphic and the other not. Such examples do not occur for $p \leqslant 2$.

Proof. (i) The last statement of (2.1) is easily deduced from the results of [2].

(ii) Suppose $p=3$ and endow $N=S^{2}$ with a metric symmetric with respect to three lines, as in Proposition 1.2. We shall obtain $M$ as a two-sheeted covering of a surface $\bar{M}$ of genus two, also symmetric with respect to three lines, by taking two copies of $\bar{M}$ and joining them along the line 1 
represented on Figure 2. $M$ is a two-sheeted unramified covering of $\bar{M}$ and by (1.6) is of genus 3. The composition of the projection with the harmonic map of Proposition (1.2) is harmonic, nonholomorphic and of degree 2.

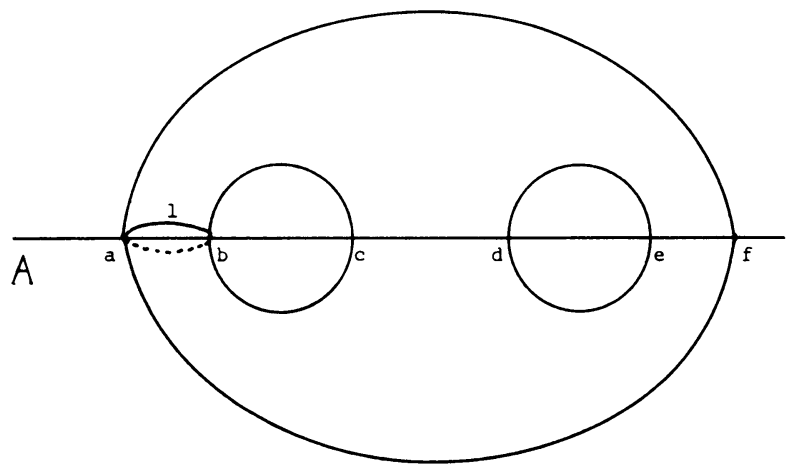

FIGURE 2

In order to build a holomorphic map of degree 2 from $M$ to $S^{2}$, we represent $M$ as the two-sheeted covering of $\bar{M}$ and define an isometry $Y$ of $M$ of order 2 as the rotation of $180^{\circ}$ around the axis $A$ (see Figure 2) which preserves each sheet, except along the line 1 where it is defined by continuity. On each sheet, the copy of the points $c, d, e$ and $f$ is fixed by $Y$, whereas the two copies of $a$ and $b$ are permuted, so that $Y$ has 8 fixed points. By Hurwitz' formula (1.6), the quotient $M / Y$ is of genus zero, and is hence conformally equivalent to the sphere with the chosen metric. The composition of the projection with that equivalence is therefore a holomorphic map of degree 2 from $M$ to $S^{2}$.

(iii) A similar example with $p=4$ is obtained by joining the two copies of $\bar{M}$ along a line with two endpoints, invariant under the rotation around $A . M$ is then of genus 4 and the rest of the construction of (ii) goes through.

(iv) For $p \geqslant 5$, we simply observe that there exists a two-sheeted covering of the surface of genus 3 built in (ii) by a surface of genus $p$. Indeed, (1.6) reduces to $2-2 p+r=-8$ or $r=2 p-10$. $r$ is therefore nonnegative and we can build the covering as above, by joining two copies of the surface of genus 3 along a closed line (if $p=5$ ) or $p-5$ segments (if $p \geqslant 6$ ).

The composition of the projection with the two maps built in (ii) gives rise to two harmonic maps of degree 4 , one holomorphic and one nonholomorphic.

3. A family of harmonic maps. In the study of surfaces, some continuous families of harmonic maps appear in a trivial way, for instance by composition of a harmonic map with continuous families of isometries or, when $M=S^{2}$, with the conformal group of $M$. These examples are taken into account in the study of the second variation of $E$ [7].

We shall now give an example of a continuous family of harmonic maps between surfaces which are not isometrically or conformally equivalent.

Let $M$ be the torus $\mathbf{R} / 2 \Pi \mathbf{Z} \times \mathbf{R} / 2 \Pi \mathbf{Z}$ endowed with its flat metric and 
Euclidean coordinates $(x, y)$. Let $0<b<a$ and denote by $N_{a, b}$ the cylinder $[-a+b, a-b] \times S^{1}$ equipped in coordinates $(u, v) \in[-a+b, a-b] \times$ $[0,2 \Pi)$ with the metric

$$
h_{\alpha \beta}=\left(\begin{array}{cc}
1 & 0 \\
0 & a^{2}-u^{2}
\end{array}\right) .
$$

This metric is $C^{\infty}$ on $N_{a, b}$ and, using a partition of unity, we can extend $N_{a, b}$ to form a $C^{\infty}$ Riemannian surface of any given genus.

We restrict our attention to maps from $M$ to $N$ whose images are in $N_{a, b}$ and which, in coordinates $(x, y)$ and $(u, v)$, are of the form $\varphi(x, y)=$ $(\Phi(x), y)$. A direct calculation shows that such a $\varphi$ is harmonic iff $d^{2} \Phi / d x^{2}+$ $\Phi=0$, that is if $\varphi(x, y)=(c \cdot \cos (x+d), y)$ with $|c| \leqslant a-b$.

When $c$ varies in $[0, a-b]$, the $\varphi$ 's form a family of harmonic maps which are not conformally equivalent. Their energy is $2 \Pi^{2} a^{2}$.

As in Remark 1.10, the torus can be replaced by a surface of higher genus, by means of a branched covering.

It is a pleasure to thank J. Eells for his constant help.

\section{REFERENCES}

1. J. Eells and J. Sampson, Harmonic mappings of Riemannian manifolds, Amer. J. Math. 86 (1964), 109-160.

2. J. Eells and J. Wood, Restrictions on harmonic maps of surfaces, Topology 15 (1976), 263-266.

3. R. C. Gunning, Lectures on Riemann surfaces, Math. Notes, no. 2, Princeton Univ. Press, Princeton, N. J., 1966.

4. L. Lemaire, Applications harmoniques de surfaces, C. R. Acad. Sci. Paris Sér. A 280 (1975), 897-899.

5. , Applications harmoniques de surfaces riemanniennes, J. Differential Geometry (to appear).

6. R. T. Smith, Harmonic mappings of spheres, Ph.D. thesis, Univ. of Warwick, England, 1972.

7. The second variation formula for harmonic mappings, Proc. Amer. Math. Soc. 47 (1975), 229-236.

8. J. C. Wood, Harmonic mappings between surfaces, Ph. D. thesis, Univ. of Warwick, England, 1974.

Department of Mathematics, University of Warwick, Coventry CV4 7AL, England

Current address: Université Libre de Bruxelles, CP 218, Campus Plaine, 1050 Bruxelles, Belgium 